\title{
Cross-section Auger analysis to study the bulk organization/structure of Mn-Co nano- composites for hybrid supercapacitors
}

Lénaïc Madec, a,d,* Céline Tang, ${ }^{\text {b,d }}$ Jean-Bernard Ledeuil, ${ }^{a}$ Domitille Giaume, ${ }^{\text {,dd }}$ Liliane GuerlouDemourgues, ${ }^{\mathrm{b}, \mathrm{d}}$ Hervé Martinez $\mathrm{z}^{\mathrm{a}, \mathrm{d}}$

${ }^{a}$ CNRS, UNIV Pau \& Pays Adour/ E2S UPPA, Institut des Sciences Analytiques et de Physicochimie pour l'Environnement et les Matériaux, UMR5254, 64000, Pau, France

${ }^{\mathrm{b}}$ CNRS, University Bordeaux, Bordeaux INP, ICMCB UMR5026, F-33600 Pessac, France

${ }^{c}$ Chimie-ParisTech, PSL Research University, CNRS Institut de Recherche de Chimie-Paris (IRCP), 75005 Paris, France

d Réseau Français sur le Stockage Electrochimique de l'Energie (RS2E), CNRS FR3459, 33 Rue Saint Leu, 80039 Amiens Cedex, France

* Corresponding Author: lenaic.madec@univ-pau.fr

\begin{abstract}
Developing new methods to prepare pseudocapacitive materials with high

pseudocapacitance/electronic conductivity is of great interest for hybrid supercapacitors.

Recently, the exfoliation/restacking of manganese and cobalt layered transition metal oxides was proposed. Despite improved electrochemical performance of such Mn-Co composites, their bulk organization (i.e. the scale at which the stacking occurs) and structure (i.e. porosity...) remains to be elucidated so far. To tackle this issue, here, SEM and Auger analysis with a nanoscale resolution, coupled to cross-section preparation is proposed. A good correlation between the restacking method, the nanoscale organization/structure of composites and resulting electrochemical performance is obtained. Importantly, the combination of cross-section with Auger analysis allows revealing the nanoscale stacking of the Mn and Co phases. Also, the porosity of the nano-composites, revealed by the cross-section preparation, is correlated to the
\end{abstract}


speed of the restacking process. A fast flocculation step forms aggregates with a porous bulk structure while a slow flocculation step leads to a dense and closed bulk structure of the aggregates. These results highlight that a better control/understanding of the organization/structure of such nano-composites can lead to further improvement. Overall, the innovative cross-section Auger approach proposed in this study should also benefit to the understanding of other nano-composites.

\section{Introduction}

Electrochemical capacitors provide relatively high power density $(10 \mathrm{~kW} / \mathrm{kg})$ at the expense of the energy $(5 \mathrm{Wh} / \mathrm{kg})$ as the charge storage reactions remain confined to the surface. ${ }^{1},{ }^{2}$ Thus, to increase the energy of commercially available carbon-based electrochemical double layer capacitors (EDLC, based on capacitive ion adsorption), the main approach is to optimize carbon/electrolyte interfaces, ${ }^{3}$ i.e. to maximize the specific surface area while controlling the pore size. ${ }^{4}$ Pseudocapacitive materials thus offer a promising alternative as they provide additional near surface redox reactions ${ }^{1}$ that allow exceeding the energy density of EDLC with, however, lower power density. ${ }^{5},{ }^{6}$ Among them, manganese oxide $\left(\mathrm{MnO}_{2}\right)^{7}$ with its high theoretical capacity (1380 F/g, considering one electron exchanged per manganese $)^{8}$ combined to a good chemical stability and a low cost, is of great interest. ${ }^{9}$ In practical conditions, capacitances in the range of $30-110 \mathrm{~F} / \mathrm{g}$ are often obtained, however, depending on the crystallographic structure. ${ }^{10}$ One of the reason is the relatively poor electronic conductivity $\left(10^{-5}\right.$ $10^{-6} \mathrm{~S} / \mathrm{cm}$ ) of $\mathrm{MnO}_{2}{ }^{8}$ that is also detrimental to the power density. 
To tackle this issue, $\mathrm{MnO}_{2}$-based nano-composites materials or nano-composites electrodes containing conductive carbons, metals, metal oxides or polymers have been successfully proposed. $.^{9},{ }^{11}$ For instance, simple $\mathrm{MnO}_{2}$ electrodeposition on Ni foam allowed obtaining capacitances higher than $400 \mathrm{~F} / \mathrm{g}$ at $1 \mathrm{~A} / \mathrm{g}$ depending on the preparation conditions. ${ }^{12,13,14}$ The drawback of this approach is, however, the relatively low $\mathrm{MnO}_{2}$ loading (about $1 \mathrm{mg} / \mathrm{cm}^{2}$ ) of the obtained electrodes. Similarly, combining $\mathrm{MnO}_{2}$ (for its high pseudocapacity) with cobalt oxyhydroxides (for their high electronic conductivities) have been shown to significantly improve the electrochemical performance. ${ }^{16},{ }^{17},{ }^{18}$ Indeed, $\mathrm{Co}_{3} \mathrm{O}_{4} @ \mathrm{MnO}_{2}$ nanowire@ nanosheetarray delivered $\sim 400 \mathrm{~F} / \mathrm{g}$ compared to $\sim 100 \mathrm{~F} / \mathrm{g}$ for $\mathrm{Co}_{3} \mathrm{O}_{4}$ nanowires at $\sim 7.5 \mathrm{~A} / \mathrm{g},{ }^{16}$ cobalt doped $(5 \%) \mathrm{MnO}_{2}$ delivered $\sim 200 \mathrm{~F} / \mathrm{g}$ compared to $\sim 100 \mathrm{~F} / \mathrm{g}$ for $\mathrm{MnO}_{2}$ at 5 A/g, ${ }^{17}$ and $\mathrm{CoO} @ \mathrm{MnO}_{2}$ nanowire@ @anosheetarray delivered $~ 600 \mathrm{~F} / \mathrm{g}$ compared to $~ 300 \mathrm{~F} / \mathrm{g}$ for CoO nanowires at 30A/g. ${ }^{18}$ However, such composite materials still lack from an optimal/controlled organization/homogeneity at the nanoscale which would further improve the electrochemical performance.

To address this challenge, an innovative synthesis approach, based on the exfoliation and restacking of manganese and cobalt layered transition metal oxides was recently proposed by some of us. ${ }^{19},{ }^{20}$ Exfoliation is achieved by weakening the interlayer bonds between metal slabs. Interestingly, as the isoelectric point (IEP) of exfoliated phases is of great importance to control and favor the nanostructuration during the restacking process, it was showed to be predictable from the metals mean oxidation state using the Multisite Complexation model (MUSIC). ${ }^{19}$ Then, by tuning the restacking process, the microstructural homogeneity of the Mn-Co composites was improved so that superior electrochemical performance were obtained compared to the starting materials, ${ }^{20}$ mostly due to the improvement of the electronic diffusion at the aggregate scale. ${ }^{21}$ 
The optimization of the Mn:Co ratio also led to further enhancement of the performance. ${ }^{21}$ These results were explained by a better organization/homogeneity of the Mn and Co phases for the exfoliated/restacked composites, as observed using scanning electron microscopy (SEM) and transmission electron microscopy (TEM) coupled to energy-dispersive X-Ray spectroscopy (EDS). ${ }^{20}$ However, SEM-EDS only probes the micro-scale while TEM-EDS involves the composites dispersion in a solvent and focuses on individual nanostructures so that it is unlikely representative of the whole sample. Note also that commonly used chemical mapping with XPS (using parallel imaging mode) has a typical spatial resolution of about 3-5 $\mu \mathrm{m}$, which is too high to study nanoscale assembly. Thus, these techniques are not suitable to observe the bulk organization (i.e. the scale at which the stacking occurs) and structure (i.e. porosity...) of nanocomposites when micrometer scale aggregates are considered. Overall, despite that these studies highlight that highly efficient pseudocapacitive materials can be obtained through this exfoliation/restacking approach, a better understanding of the bulk organization/structure of such composites at the nanoscale remains a challenge so far.

To tackle this issue, this study proposes Auger analysis with a nanoscale resolution coupled to a cross-section preparation as a powerful approach to elucidate the bulk organization/structure of Mn-Co composites. To the best of our knowledge, this approach has never been used for such composite materials. Results are discussed regarding both structural and electrochemical properties of the composites.

\section{Material and methods}


Synthesis - The preparation of layered proton-inserted birnessite manganese oxide $\left(\mathrm{H}-\mathrm{MnO}_{2}\right)$ and layered $\beta(\mathrm{III})$-type cobalt oxyhydroxide ( $\beta 3-\mathrm{CoOOH})$ are fully described elsewhere. ${ }^{20}$ The exfoliation of $\mathrm{H}-\mathrm{MnO}_{2}$ and $\beta 3-\mathrm{CoOOH}$ was performed in tetrabutylammonium hydroxide $(\mathrm{TBAOH})$ solutions for about 2 weeks at room temperature under vigorous stirring, allowing $\mathrm{TBA}^{+}$ions intercalation via proton exchange. For $\mathrm{H}-\mathrm{MnO}_{2}$ and $\beta 3-\mathrm{CoOOH}$, the optimized molar ratio of $\mathrm{TBA}^{+} / \mathrm{H}^{+}$was fixed to 10 and 2 , respectively. Resulting colloidal suspensions were then centrifugated at $6000 \mathrm{rpm}$ for $15 \mathrm{~min}$ to remove any unexfoliated materials. Note that the stability of these colloidal suspensions (at $\mathrm{pH}=12$, i.e. negatively charged) were up to few months. Then, based on the determined IEP, ${ }^{19}$ the restacking of the as-prepared exfoliated Mnand Co-based nanosheets to form Mn-Co composites was then performed using two methods. For better clarity, Figure 1 shows a schematic of the different approaches used to prepare the composites. Method 1: the two stable colloidal suspensions (i.e. at $\mathrm{pH}=12$ ) were mixed then floculation was induced by the fast (1s) or slow (2 hours) addition into a nitric acid solution to lower the $\mathrm{pH}$ to 4.5 so that exfoliated/restacked composites RMC1a and RMC1b were obtained, respectively. Method 2: the $\mathrm{pH}$ of the two stable colloidal suspensions (i.e. $\mathrm{pH}=12$ ) was lowered separetely to 4.5 by addition of a nitric acid solution. As this step can destabilize the suspensions to form aggregates due to the increase of the ionic strength, 3 centrifugation/ sonication cycles were performed to counteract this effect. Floculation was then induced by mixing the two suspensions with a control of the $\mathrm{pH}$ so that exfoliated/restacked composites RMC2 was obtained. Materials were then centrifugated and dried. For comparison, a reference sample was also prepared by mechanical grinding (MG), in a mortar, of the $\mathrm{H}-\mathrm{MnO} 2$ and $\beta 3-\mathrm{CoOOH}$ powders. Note that for all mixed Mn-Co composites, Mn:Co ratio was fixed to 1:1. 


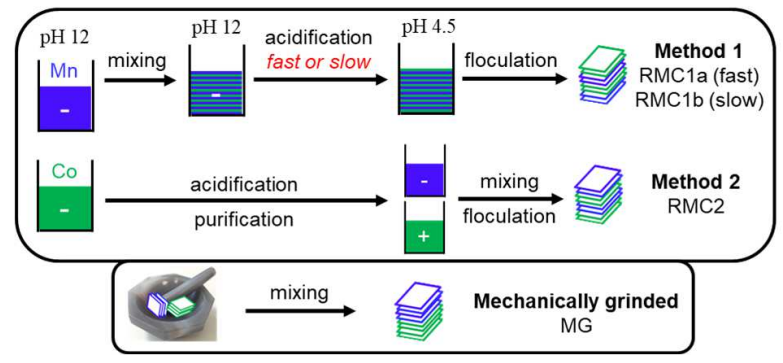

Figure 1. Schematic of the different approaches used in this work to prepare the Mn-Co composites.

Structural and electrochemical properties - The average coherent domain size (nm) along the slab stacking direction and the specific BET surface area $\left(\mathrm{m}^{2} / \mathrm{g}\right)$ of $\mathrm{H}-\mathrm{MnO}_{2}, \beta 3-\mathrm{CoOOH}$ and Mn-Co composites were determined following previously described X-ray diffraction/Scherrer method and $\mathrm{N}_{2}$ adsorption analysis conditions, respectively. ${ }^{20}$ For clarity, diffraction patterns were recorded with a $0.0167^{\circ}(2 \theta)$ step size and a $2.122^{\circ}(2 \theta)$ active width in the detector using a Philips Panalytical X'Pert Pro diffractometer with a Bragg-Brentano $\theta-\theta$ geometry. Copper K $\alpha$ radiation $\left(\lambda_{\mathrm{K} \alpha 2}=1.54439 \AA, \lambda_{\mathrm{K} \alpha 1}=1.54056 \AA\right)$ and cobalt $\mathrm{K} \alpha$ radiation $\left(\lambda_{\mathrm{K} \alpha 2}=1.7928 \AA, \lambda_{\mathrm{K} \alpha 1}=\right.$ $1.7889 \AA$ A) were used for manganese oxides and cobalt oxyhydroxides powders, respectively. Electrochemical performance were evaluated in a three-electrodes cell using $0.5 \mathrm{M} \mathrm{K}_{2} \mathrm{SO}_{4}$ as electrolyte with platinum and $\mathrm{Ag} / \mathrm{AgCl}$ as counter and reference electrodes. Working electrodes were prepared by mixing $80 \mathrm{wt} . \%$ of active material with $5 \mathrm{wt} . \%$ PTFE (Sigma-Aldrich, $60 \mathrm{wt} . \%$ water suspension) and 15 wt.\% acetylene black (100\% compressed, Alfa Aesar) with ethanol. 8 mm diameter discs were cut from the obtained self-supported film and pressed on stainless steel grid at $6 \mathrm{t} / \mathrm{cm}^{2}$. Cyclic voltammetry was performed using a VMP3 potentiostat (Biologic)/ECLab software between $0-0.8 \mathrm{~V}$ from 0.5 to $500 \mathrm{mV} / \mathrm{s}$. 
Mn-Co composites cross-section preparation and Scanning electron microscopy (SEM), Auger electron spectroscopy (AES) and scanning Auger microscopy (SAM) analysis - Cross-section samples were prepared using a JEOL Cross-Polisher (JEOL Ltd, Tokyo, Japan) in a nitrogenfilled glove box. Powder samples were hand-mixed in a silver conducting epoxy resin then deposited on a silicon wafer. Resulting assemblies were then exposed (from the silicon wafer side and at normal angle) to an $\mathrm{Ar}^{+}$ion beam $(6 \mathrm{keV}, \sim 120 \mu \mathrm{A})$ for $4 \mathrm{~h}$ at $1.10^{-4} \mathrm{~Pa}$. More details about the technique can be found in previous works. ${ }^{22},{ }^{23}$ This allowed obtaining perfect planar surfaces with no $\mathrm{Ar}^{+}$ion implantation (Ar LMM Auger lines were never observed at the kinetic energies of 211 and $195 \mathrm{eV}$ ). Thus, possible sample damage would be limited to amorphization phenomenon with the same chemical composition. ${ }^{24}{ }^{25},{ }^{26}$ For clarity, Figure S1 shows typical SEM images of a MnCo composite cross-section so that readers can appreciate the flatness of the cross-section as well as the resulting assembly using a silicon wafer and a silver epoxy resin.

SEM, AES and SAM analysis were performed using a JEOL JAMP $9500 \mathrm{~F}$ Auger spectrometer (JEOL Ltd, Tokyo, Japan) equipped with a Schottky Field Emission gun and a hemi-spherical analyser coupled with a high dynamic multichannel detector following a previously described procedure $.{ }^{23},{ }^{27}$ For clarity, considering the beam energy/probe current used (10 keV/5 nA) for Auger analysis, the probe size for AES (i.e. to record an Auger spectrum) is about $20 \mathrm{~nm}$ leading to a spatial resolution for SAM (i.e. to record an Auger image) of about $30 \mathrm{~nm}$. Also, the probing depth is about $3 \mathrm{~nm}$. Thus, the simultaneous presence of Mn and Co transitions in an Auger spectrum means that the $\mathrm{Mn}$ and Co containing phases are located together at a $20 \mathrm{~nm}$ wide scale and for a $3 \mathrm{~nm}$ depth. The operating pressure was $<2 \times 10^{-7} \mathrm{~Pa}$ and analysis were performed at $30^{\circ}$ tilt to prevent charging effect. AES survey spectra were recorded between 15 to $2000 \mathrm{eV}$ with $1 \mathrm{eV}$ step size using a focused probe $(10 \mathrm{keV} / 5 \mathrm{nA}$, i.e. a probe size of about $20 \mathrm{~nm})$ and a 
CRR mode (constant relative resolution, also called FRR mode) corresponding to a relative energy resolution $(\mathrm{dE} / \mathrm{E}=$ constant) with $\mathrm{dE} / \mathrm{E}=0.5 \%$ (high sensitivity). Scanning Auger microscopy (SAM, elemental 2D distribution) images were recorded using a CAE mode (constant analyzer energy, also called FAT or CAR mode) corresponding to a fixed energy resolution ( $\mathrm{dE}=$ constant). Images are represented using "peak minus background" $(\mathrm{P}-\mathrm{B})$ Auger intensity for a transition $(\mathrm{dE} / \mathrm{E}=0.5 \%)$. The spatial resolution was about $30 \mathrm{~nm}$ (at $10 \mathrm{keV} / 5 \mathrm{nA})$. Also, for SAM analysis, an "auto probe tracking" correction was applied to control and compensate any potential drift.

\section{Results and discussion}

Previous XRD analysis ${ }^{20}$ showed that both the mechanically grinded composite and the exfoliated/restacked composites showed no structural change in the layers, i.e. very close diffractograms compared to the initial $\mathrm{H}-\mathrm{MnO}_{2}$ and $\beta 3-\mathrm{CoOOH}$ phases. ${ }^{20}$ Table 1 shows the average coherent domains size (nm) along the slab stacking direction and the specific BET surface area $\left(\mathrm{m}^{2} / \mathrm{g}\right)$ for all composites. The thickness of Mn-based domains similarly decreased regardless of the restack procedure, while the thickness of Co-based domains did not change except for RMC1a so that an increase of the specific BET surface area can be expected. However, RMC1a and RMC2 gave intermediate BET surface area (between 90 to $100 \mathrm{~m} 2 / \mathrm{g}$ ) compared to the starting $\mathrm{H}-\mathrm{MnO}_{2}\left(85 \mathrm{~m}^{2} / \mathrm{g}\right)$ and $\beta 3-\mathrm{CoOOH}\left(110 \mathrm{~m}^{2} / \mathrm{g}\right)$ materials while a drastic decrease was observed for RMC1b $\left(2 \mathrm{~m}^{2} / \mathrm{g}\right)$. Regarding the mechanically grinded (MG) composite, no significant change was observed compared to the starting materials so that the efficiency of the mixing step is expected to be rather poor. Overall, these results suggest: (i) an 
homogeneous organization with a porous structure for the RMC1a and RMC2 composites due to the fast flocculation step of oppositely charged Mn- and Co- nanosheets during the restacking process and (ii) a much denser structure for the RMC1b composite due to the slow flocculation step during the restacking process. At this point, however, the formation of nano-composites can not be proven by XRD and thus their bulk organization/structure still need to be investigated.

Table 1. Average coherent domain sizes (nm) along the slab stacking direction (i.e. thickness) of the Mn- and Cophases for the starting materials $\left(\mathrm{H}-\mathrm{MnO}_{2}\right.$ and $\left.\beta 3-\mathrm{CoOOH}\right)$, the mechanically grinded composite and the exfoliated/restacked Mn-Co composites as determined by the Scherrer method and their specific BET surface areas.

\begin{tabular}{|c|c|c|c|}
\hline \multirow[b]{2}{*}{ Sample } & \multicolumn{2}{|c|}{ Size of coherent domain (nm) } & \multirow[b]{2}{*}{$\begin{array}{c}\text { Specific BET } \\
\text { surface }\left(\mathrm{m}^{2} / \mathrm{g}\right)\end{array}$} \\
\hline & $\begin{array}{c}\text { (001) line of } \\
\text { Mn-based phase }\end{array}$ & $\begin{array}{c}(003) \text { line of } \\
\text { Co-based phase }\end{array}$ & \\
\hline $\mathrm{H}-\mathrm{MnO}_{2}$ & 11 & - & 85 \\
\hline$\beta 3-\mathrm{CoOOH}$ & - & 5 & 110 \\
\hline MG & 12 & 3 & 95 \\
\hline RMC1a & 7 & 3 & 92 \\
\hline RMC1b & 7 & 6 & 2 \\
\hline RMC2 & 6 & 6 & 100 \\
\hline
\end{tabular}

To tackle this issue, SEM, AES and SAM analysis were performed on cross-section of all composites. SEM and SAM (elemental 2D distribution, from Mn LMM and Co LMM transitions, in blue and green, respectively) images and Auger spectra are shown in Figure 2 for the mechanically grinded (MG) composite and in Figure 3 for the exfoliated/restacked Mn-Co (RMC1a, RMC1b and RMC2) composites. Overall, SEM images showed micrometer scale aggregates for all composites (Figures 2-3, S2-5). The bulk structure of the aggregates was clearly different, however, as function of the composite preparation method. SEM images taken at different magnification showed a porous bulk structure for both RMC1a and RMC2 while a much denser and closed bulk structure was observed for RMC1b (Figures 3, S3 and S5). This 
result explains the very low specific BET surface area of RMC1b $\left(2 \mathrm{~m}^{2} / \mathrm{g}\right)$ compared to RMC1a and RMC2 (about $95 \mathrm{~m}^{2} / \mathrm{g}$ ). It also highlights that the flocculation step speed of oppositely charged Mn- and Co- nanosheets controls the porosity of the nano-composites. The fast decrease of the $\mathrm{pH}$ to induce the flocculation leads to a porous bulk structure of the aggregates (RMC1a and $\mathrm{RMC2}$ ) while the slow decrease of the $\mathrm{pH}$ leads to a dense and closed bulk structure (RMC1b). Overall, SEM analysis coupled to cross-section preparation allowed revealing the bulk structure of Mn-Co composites, highlighting the interest of this approach compared to classical SEM.

Now, considering that the average coherent domain sizes of both Mn and Co phases along the slab stacking direction (i.e. their thickness) is lower than $10 \mathrm{~nm}$ (Table 1), the aggregates are expected to be a stacking of $\mathrm{Mn}$ and Co phases. The bulk organization (i.e. the scale at which the stacking occurs) of the composites was then investigated. At this point, conventional approaches such as SEM-EDS only probes the micro-scale while TEM-EDS involves the composite dispersion in a solvent and the study of individual nanostructures, which is unlikely representative of the whole sample. Instead, cross-section Auger analysis was proposed as an alternative. It is reminded that the probing depth is about $3 \mathrm{~nm}$, the probe size about $20 \mathrm{~nm}$ (to record an Auger spectrum) and the spatial resolution of about $30 \mathrm{~nm}$ (to record an Auger image). In Auger spectra, transitions were observed at about 500 and $480 \mathrm{eV}$ for the $\mathrm{O} \mathrm{KLL}$, at about 630, 580 and $530 \mathrm{eV}$ (the latter overlapped with the $\mathrm{O}$ KLL at $500 \mathrm{eV}$ ) for the Mn LMM and at about 775, 710 and $645 \mathrm{eV}$ (the latter overlapped with the Mn LMM at $630 \mathrm{eV}$ ) for the Co LMM. For the MG composite (Figure 2), Auger spectra taken at different positions of the aggregates showed either Mn LMM or Co LMM transitions. Results for additional aggregates shown in Figure S2 provides evidence that this result is representative of the whole sample. 
Thus, the use of the mechanical grinding approach leads to a microscale segregated composite, as expected. At the opposite, aggregates of exfoliated/restacked composites showed the simultaneous presence of Mn and Co transitions in most Auger spectra (Figure 3) for a probe size of about $20 \mathrm{~nm}$ (with a $3 \mathrm{~nm}$ probing depth). This result supported by the analysis of additional aggregates (Figures S3-5) highlights that the stacking of Mn and Co phases occurred at the nanoscale. Therefore, these composites can be denoted as nano-composites, which could not be proven by XRD analysis. Moreover, some discrepancies were observed as function of the restacking method. In the case of RMC2 (prepared by method 2, Figure 1), some of the Auger spectra showed either Mn or Co transitions while for RMC1a and RMC1b (prepared by method 1, Figure 1), all Auger spectra showed both Mn and Co transitions (Figures 3, S3-5). Therefore, RMC1a and RMC1b are more homogeneously stacked at the nanoscale compared to RMC2. This is further confirmed by SAM images (Figures 3, S3-S5) that showed a more homogeneous distribution of the Mn and Co areas (in blue and green, respectively) for RMC1a and RMC1b compared to a more segregated distribution of the Mn and Co areas for RMC2. This is explained by the restacking method used. For RMC1a and RMC1b, colloidal suspensions of Mn and Co nanosheets were mixed together before flocculation (i.e. before the $\mathrm{pH}$ was lowered) so that a better nanoscale stacking is obtained. For RMC2, the $\mathrm{pH}$ of the separate colloidal suspensions were first lowered before mixing, favoring the formation of aggregates due to the destabilization of the solutions. Overall, Auger analysis coupled to cross-section preparation allowed elucidating the bulk organization (i.e. the scale at which the stacking occurs) of Mn-Co composites, which remained a challenge so far. 

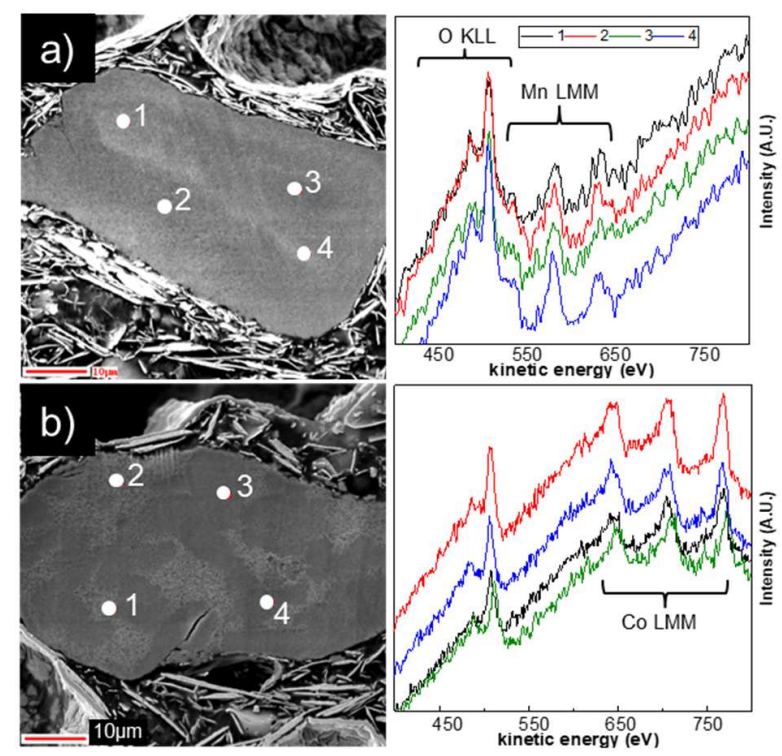

Figure 2. SEM images for the mechanically grinded (MG) composite and Auger spectra taken at different positions as indicated.

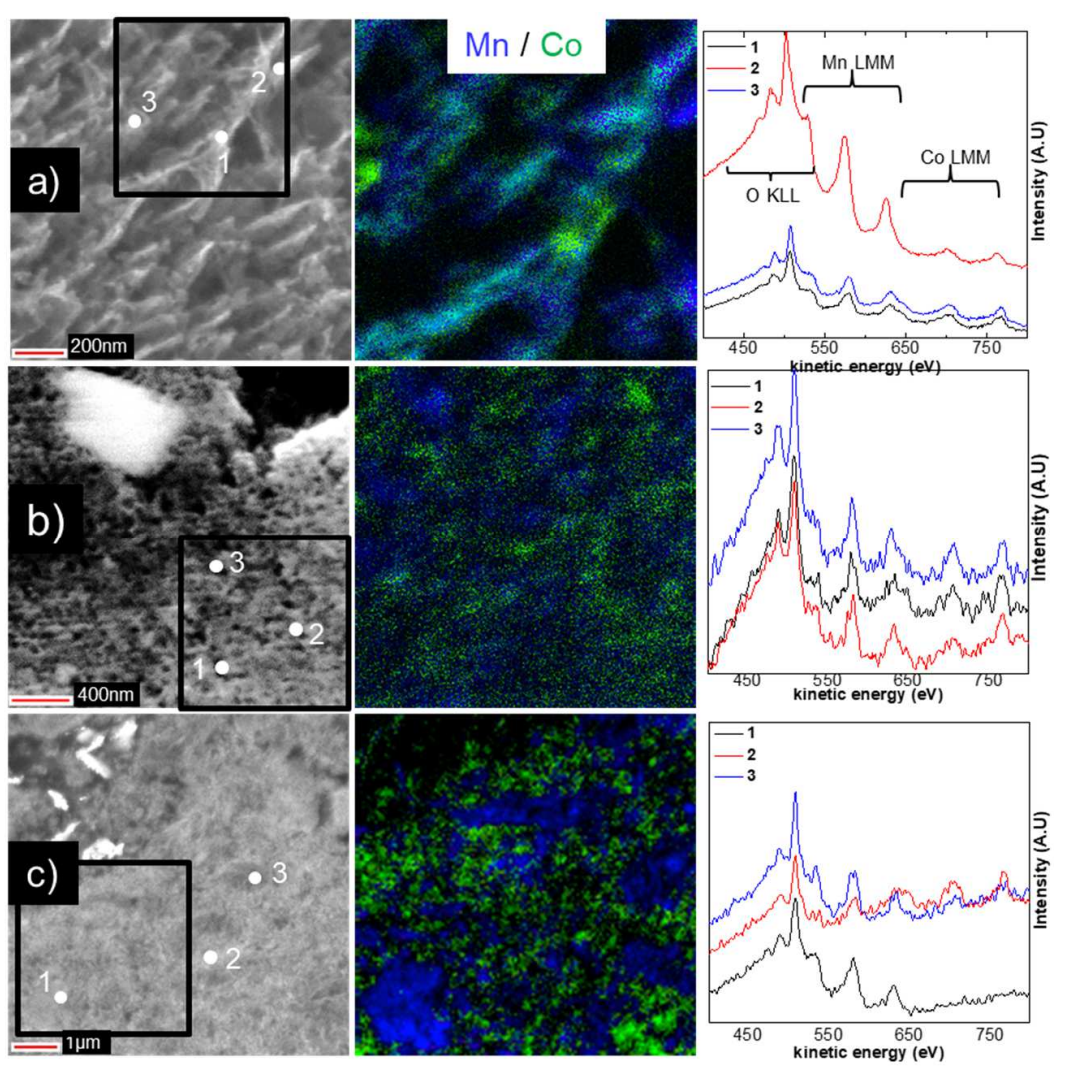

Figure 3. SEM and SAM images (elemental 2D distribution taken at the indicated areas, from Mn LMM and Co LMM transitions, in blue and green, respectively as well as Auger spectra taken at indicated positions for the 
exfoliated/restacked Mn-Co nano-composites a) RMC1a, b) RMC1b and c) RMC2. Note that the white/grey flakes observed in the SEM images correspond to the silver epoxy resin used for sample preparation. ${ }^{23}$

Based on these results, the electrochemical performance of all composites (Figure 4) can be better understood. For comparison, the theoretical capacitance calculated on the basis of the starting $\mathrm{H}-\mathrm{MnO}_{2}$ and $\beta 3-\mathrm{CoOOH}$ capacitances (with the 1:1 ratio) is also shown in Figure 4 (dashed line). For the MG composite, slightly improved performance were observed compared to the theoretical capacitance, indicating the beneficial impact of the Co oxyhydroxide addition to the $\mathrm{MnO}_{2}$ birnessite. For the nano-composites RMC1a and RMC2, higher improvements were observed. In particular, RMC2 showed superior capacitances compared to MG, whatever the scan rate was. For RMC1a, higher capacitances than $\mathrm{MG}$ were observed but up to a scan rate of about $50 \mathrm{mV} / \mathrm{s}$. These results can be explained by a better electronic conductivity due to the homogeneous stacking of $\mathrm{MnO}_{2}$ birnessite with highly electronic conductive Co oxyhydroxide at the nanoscale. This thus leads to a better use of the birnessite pseudocapacitance in the nanocomposites, in agreement with the more rectangular shapes of the CV curves previously observed compared to the starting $\mathrm{H}-\mathrm{MnO}_{2} \cdot{ }^{20}$ Interestingly, despite a homogeneous nanoscale stacking, RMC1b showed poor performance due to its denser and closed bulk structure (i.e. low BET surface area) as observed by cross-section SEM. 


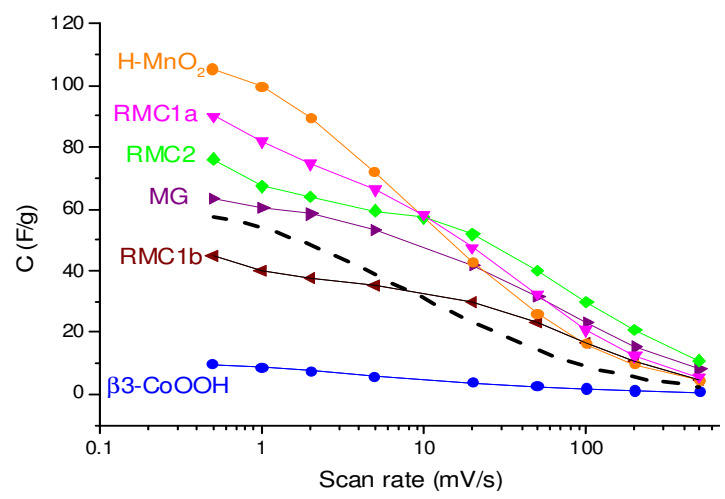

Figure 4. Variation of specific capacitance (F/gactive-material) vs. scan rate (mV/s) obtained for the starting materials $\left(\mathrm{H}-\mathrm{MnO}_{2}\right.$ and $\left.\beta 3-\mathrm{CoOOH}\right)$, the mechanically grinded composite (MG) and the exfoliated/restacked $\mathrm{Mn}-\mathrm{Co}$ nano-composites (RMC1a, b and RMC2). The dashed line corresponds to the theoretical average capacitance calculated on the basis of the capacitances of $\mathrm{H}-\mathrm{MnO}_{2}$ and $\beta 3-\mathrm{CoOOH}$, for the $1: 1$ ratio.

\section{Conclusions}

In this study, the bulk organization (i.e. the scale at which the stacking occurs) and structure (i.e. porosity...) of Mn-Co composites formed by different exfoliation/restacking methods was investigated. XRD analysis as well as previous SEM- and TEM-EDS could not prove, however, the formation of nano-composites. To tackle this issue, SEM and Auger analysis coupled to cross-section preparation was proposed for the time to study such composites. Importantly, cross-section Auger analysis allowed revealing that the stacking of Mn and Co phases occurred at the nanoscale, which could not be proven so far. In addition, the porosity of the nanocomposites was revealed by the cross-section preparation and explained the low BET surface area of one of the sample. Interestingly, it highlighted that a fast flocculation step forms aggregates with a porous bulk structure while a slow flocculation step leads to a dense and closed bulk structure of the aggregates. Overall, a very good correlation between the restacking methods used, the nanoscale organization/structure of the composites and the resulting electrochemical 
performance was obtained. These results thus open new possibility to improve further the performance of such nano-composites for hybrid supercapacitors. Finally, the innovative crosssection Auger approach proposed in this study should also benefit to other researchers working on understanding and improving other nano-composites.

\section{Acknowledgment}

This research was performed in the framework of "Réseau sur le Stockage Electrochimique de l'Energie" (RS2E) and the ANR program no. ANR-10-LABX-76-01.

\section{References}

1. B. E. Conway, Electrochemical Supercapacitors: Scientific Fundamentals and Technological Applications, Kluwer, New York, (1999).

2. P. Simon and Y. Gogotsi, Nat. Mater., 7, 845-854 (2008).

3. P. Simon and Y. Gogotsi, Acc. Chem. Res., 46, 1094-1103 (2013).

4. J. Chmiola, G. Yushin, Y. Gogotsi, C. Portet, P. Simon, and P. L. Taberna, Science (80-. )., 313, 1760-1763 (2006).

5. L. L. Zhang, R. Zhou, and X. S. Zhao, J. Mater. Chem., 20, 5983-5992 (2010).

6. V. Augustyn, P. Simon, and B. Dunn, Energy Environ. Sci., 7, 1597-1614 (2014).

7. H. Y. Lee and J. B. Goodenough, J. Solid State Chem., 144, 220-223 (1999). 
8. M. Toupin, T. Brousse, and D. Bélanger, Chem. Mater., 16, 3184-3190 (2004).

9. W. Wei, X. Cui, W. Chen, and D. G. Ivey, Chem. Soc. Rev., 40, 1697-1721 (2011).

10. O. Ghodbane, J. L. Pascal, and F. Favier, ACS Appl. Mater. Interfaces, 1, 1130-1139 (2009).

11. J. G. Wang, F. Kang, and B. Wei, Prog. Mater. Sci., 74, 51-124 (2015) http://dx.doi.org/10.1016/j.pmatsci.2015.04.003.

12. M. Zhang, Y. Chen, D. Yang, and J. Li, J. Energy Storage, 29, 101363 (2020).

13. M. Zhang, D. Yang, and J. Li, J. Energy Storage, 30, 101511 (2020).

14. M. Zhang, D. Yang, and J. Li, Vacuum, 178, 109455 (2020).

15. M. Toupin, T. Brousse, and D. Bélanger, Chem. Mater., 16, 3184-3190 (2004).

16. J. Liu, J. Jiang, C. Cheng, H. Li, J. Zhang, H. Gong, and H. J. Fan, Adv. Mater., 23, 2076-2081 (2011).

17. B. C. Kim, C. Justin Raj, W. J. Cho, W. G. Lee, H. T. Jeong, and K. H. Yu, J. Alloys Compd., 617, 491-497 (2014) http://dx.doi.org/10.1016/j.jallcom.2014.08.018.

18. X. Wang, Y. Xiao, D. Su, L. Zhou, S. Wu, L. Han, S. Fang, and S. Cao, Electrochim. Acta, 194, 377-384 (2016).

19. C. Tang, D. Giaume, L. Guerlou-Demourgues, G. Lefèvre, and P. Barboux, Langmuir, 34, 6670-6677 (2018).

20. C. Tang, D. Giaume, F. Weill, N. Penin, M. A. Dourges, H. Saadaoui, and L. GuerlouDemourgues, ACS Appl. Energy Mater. (2019). 
21. C. Tang, thesis, niversité de Bordeaux, 2 (2017) https://tel.archives-ouvertes.fr/tel01696266/document.

22. A. Uhart, J. B. Ledeuil, B. Pecquenard, F. Le Cras, M. Proust, and H. Martinez, ACS Appl. Mater. Interfaces, 9, 33238-33249 (2017).

23. L. Madec, J.-B. Ledeuil, G. Coquil, L. Monconduit, and H. Martinez, ACS Appl. Energy Mater., doi/10.1021/acsaem.9b01115 (2019) http://pubs.acs.org/doi/10.1021/acsaem.9b01115.

24. T. Schuhrke, M. Mändl, J. Zweck, and H. Hoffmann, Ultramicroscopy, 41, 429-433 (1992).

25. J. P. McCaffrey, M. W. Phaneuf, and L. D. Madsen, Ultramicroscopy, 87, 97-104 (2001).

26. M. Mitome, J. Electron Microsc. (Tokyo)., 62, 321-326 (2013).

27. L. Madec, J. B. Ledeuil, G. Coquil, G. Gachot, L. Monconduit, and H. Martinez, J. Power Sources, 441 (2019). 


\section{Graphical abstract}

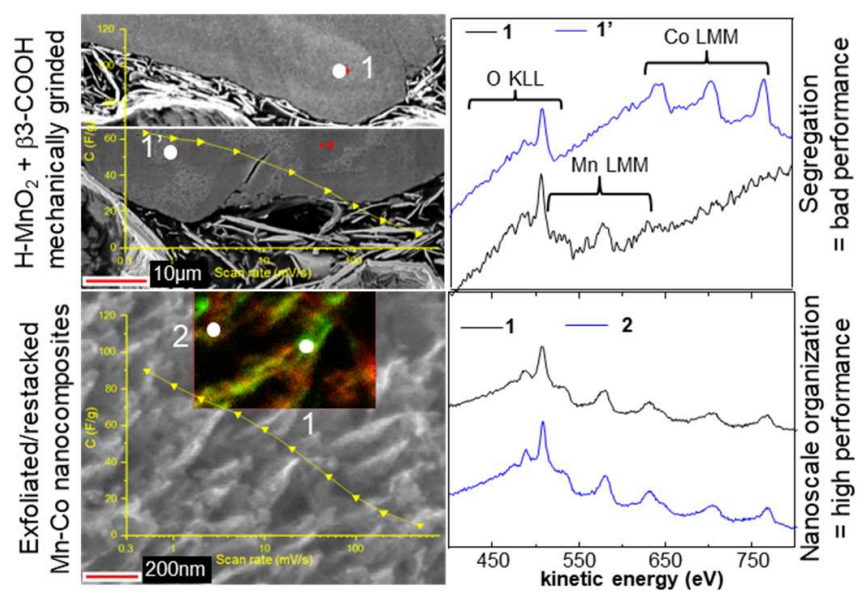

